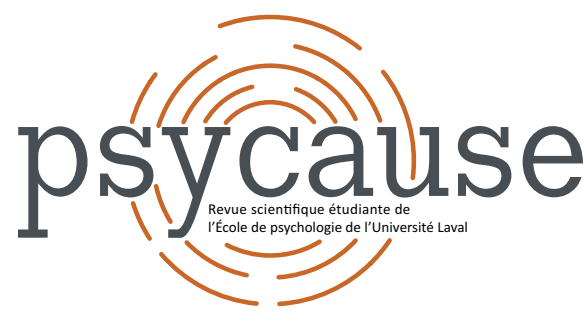

\section{PSYCAUSE}

Revue scientifique étudiante de l'École de psychologie de l'Université Laval

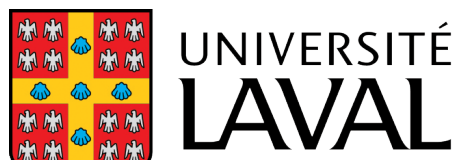

Faculté des sciences sociales École de psychologie

\title{
LIEN ENTRE LE STRESS PARENTAL ET LA SENSIBILITÉ MATERNELLE ENTRE SIX ET 18 MOIS
}

Catherine POVEDA PERDOMO ${ }^{1,4, *}$, Claudia BERGERON ${ }^{1,4}$, Mathilde YERGEAU ${ }^{1,4}$,

Laura-Émilie SAVAGE ${ }^{1,4}$, Audrey GAUTHIER-LÉGARÉ ${ }^{1,4}$, Jean-Pascal LEMELIN ${ }^{3,4}$, Marc PROVOST² et George M. TARABULSY',4

${ }^{1}$ École de psychologie, Université Laval

2 Département de psychologie, Université du Québec à Trois-Rivières

${ }^{3}$ Faculté d'éducation, Université de Sherbrooke

${ }^{4}$ Centre de recherche universitaire sur les jeunes et les familles (CRUJeF)

* catherine.poveda-perdomo.1@ulaval.ca

\section{Pour citer l'article}

Poveda Perdomo, C., Bergeron, C., Yergeau, M., Savage, L.-É., Gauthier-Légaré, A., Lemelin, J.-P., Provost, M., \& Tarabulsy, G. M. (2019). Lien entre le stress parental et la sensibilité maternelle entre six et 18 mois. Psycause: Revue scientifique étudiante de l'École de psychologie de l'Université Laval, 9(2), 19-20. 
Nesteruk, O., Helmstetter, N.-M., Gramescu, A., Siyam, M. H., \& Price, C. A. (2015). Development of ethnic identity in young adults from immigrant families: "I want to hold onto my roots, but I also want to experience new routes". Marriage \& Family Review, 51(5), 466 487. doi:10.1080/01494 929.2015.1060288
Phinney, J. S., Romero, I., Nava, M., \& Huang, D. (2001). The role of language, parents, and peers in ethnic identity among adolescents in immigrant families. Journal of Youth and Adolescence, 30(2), 135 153. doi:10.1023/A:1010389607319

\section{Pour citer l'article}

Miljus, M., Montreuil, F., Garceau, L., Taing, J., \& Yampolsky, M. A. (2019). Langue d'origine et expérience identitaire chez les Canadiens de deuxième génération. Psycause: Revue scientifique étudiante de l'École de psychologie de l'Université Laval, 9(2), 17-19.

\section{LIEN ENTRE LE STRESS PARENTAL ET LA SENSIBILITÉ MATERNELLE ENTRE SIX ET 18 MOIS}

Catherine POVEDA PERDOMO ${ }^{1,4, *}$, Claudia BERGERON ${ }^{1,4}$, Mathilde YERGEAU ${ }^{1,4}$, Laura-Émilie SAVAGE ${ }^{1,4}$, Audrey GAUTHIER-LÉGARÉ ${ }^{1,4}$, Jean-Pascal LEMELIN ${ }^{3,4}$, Marc PROVOST ${ }^{2}$ et George M. TARABULSY1,4

${ }^{1}$ École de psychologie, Université Laval; ${ }^{2}$ Département de psychologie, Université du Québec à Trois-Rivières; ${ }^{3}$ Faculté d'éducation, Université de Sherbrooke; ${ }^{4}$ Centre de recherche universitaire sur les jeunes et les familles (CRUJeF)

* catherine.poveda-perdomo.1@ulaval.ca

\section{Mots-clés : Sensibilité maternelle; Stress parental; Développement de l'enfant}

La sensibilité maternelle (SM) réfère à l'habileté de la mère à percevoir et à interpréter les signaux de son enfant, ses comportements et ses émotions, ainsi que d'y répondre rapidement et de façon appropriée (Ainsworth, 1982; Neuhauser, 2018). La SM est un des plus importants médiateurs postnatals du développement, puisqu'elle est associée à différentes sphères du développement de l'enfant (Tarabulsy, Moran, Pederson, Provost \& Larose, 2011). Plusieurs études identifient le stress parental (SP) comme étant un important corrélat de la SM. Le SP se définit comme l'écart ressenti par le parent, d'une part entre les exigences posées par la situation de la parentalité et par l'enfant lui-même, et d'autre part, par les ressources et les habiletés disponibles pour répondre à ces exigences (Lacharité, Ethier \& Couture, 1996). La plupart des études démontrent que le SP et la SM sont inversement corrélés (c.-à-d. que le fait de vivre plus de SP est lié à une baisse de la SM; Neuhauser, 2018). Il est possible qu'une SM moindre engendre des situations de stress pour les parents. Or, la plupart des travaux utilisent des devis transversaux et ne permettent donc pas de connaître la direction du lien (Booth \& coll., 2018). Cette étude a pour premier objectif d'examiner les liens entre le $\mathrm{SP}$ et la SM lors d'interactions mère-enfant à six, 10, 15 et 18 mois. Le deuxième objectif est de vérifier les liens longitudinaux entre les concepts, en tenant compte de la contribution des variables au temps précédent.

\section{Méthode}

Les données pour les quatre temps de mesures sont recueillies auprès de 83 dyades et les analyses sont effectuées sur les 160 dyades recrutées avec la méthode de traitement des données manquantes du maximum de vraisemblance. L'échantillon d'enfants comprend 89 filles et 71 garçons. Le SP est évalué à l'aide du Parenting Stress Index (PSI - short form, Abidin, 1995) qui mesure la détresse parentale, les interactions dysfonctionnelles et les difficultés du nourrisson. La SM est évaluée à l'aide du Maternal Behavior Q-set (MBOS; Pederson \& Moran, 1995), une mesure observationnelle de ce construit. Des rencontres à la maison ont été réalisées lorsque les enfants étaient âgés de six, 10,15 et 18 mois pour compléter ces deux mesures.

\section{Résultats et discussion}

Concernant le premier objectif, des corrélations de Pearson montrent que contrairement à ce que la littérature rapporte, le SP et la SM ont une très grande stabilité et que leur évolution dans le temps semble indépendante l'une de l'autre, particulièrement à six et 10 mois, où les concepts ne sont pas liés entre eux. Des corrélations modérées apparaissent à 15 et 18 mois et pourraient être expliquées par des changements dans les demandes de l'enfant, dans son développement ou dans l'écologie développementale durant sa deuxième année de vie. Cependant, le fait saillant de 
ces résultats concerne la force des liens entre les différents temps de mesure pour une même variable et que, cette stabilité diminue la variance commune avec l'autre concept. Ces résultats indiquent que des facteurs stables puissent expliquer l'évaluation du SP par le parent et la qualité de la SM, tels que les traits de personnalité, les antécédents développementaux et les modes relationnels des parents.

Quant au second objectif, un modèle de corrélations croisées (crosslag) a été exploré par le biais d'analyses acheminatoires. La Figure 1 montre qu'un seul lien longitudinal émerge, soit entre le SP à 10 mois et la SM à 18 mois. Ainsi, le SP n'apporte pas une contribution significative à la SM au temps suivant lorsqu'on considère la contribution de la SM du temps précédent, et ce pour tous les autres temps de mesures du modèle. Ce lien indique que le SP augmente à 10 mois, mais aurait un effet sur la SM seulement à l'âge de 18 mois. Ce gain de SP pourrait être attribuable au développement moteur de l'enfant ou pourrait coïncider avec la fin du congé de maternité. La diminution de la SM à 18 mois pourrait s'expliquer par le fait que l'enfant devient plus autonome et exprime mieux ses besoins, ce qui demanderait moins d'attention de la mère.

Le devis longitudinal de cette étude représente sa principale force. II a permis de mettre en lumière que contrairement à ce qui est véhiculé dans la littérature scientifique, les liens entre la SM et le SP sont moins importants lorsqu'on tient compte de la contribution des variables au temps précédent et qu'on les évalue de façon longitudinale. Ces résultats démontrent l'importance de mieux comprendre l'interaction entre ces concepts et de mieux connaître leur évolution dans le temps.

\section{Références}

Abidin, R. R. (1995). Parenting stress index: Professional manual (3rd ed.). Odessa, FL: Psychological Assessment Resources.

Ainsworth, M. D. (1982). Attachment: Retrospect and prospect. New-York: Basic books.

Booth, A. T., Macdonald, J. A., \& Youssef, G. J. (2018). Contextual stress and maternal sensitivity: A meta-analytic review of stress associations with the Maternal Behavior Q-Sort in observational studies. Developmental Review, 48, 145177. doi: 10.1016/j.dr.2018.02.002

Lacharité, C., Ethier, L., \& Couture, G. (1996). The influence of partners on parental stress of neglectful mothers. Child Abuse Review, 5(1), 18-33. doi:10.1002/(SICI)10990852(199603)5:1<18: :AID-CAR218>3.0.CO;2-Z

Neuhauser, A. (2018). Predictors of maternal sensitivity in at-risk families. Early Child Development and Care, 188(2), 126142. doi:10.1080/03004430.2016.1207065

Pederson, D. R., \& Moran, G. (1995). A categorical description of infant-mother relationships in the home and its relation to Q-sort measures of infant-mother interaction. Monographs of the Society for Research in Child Development, 60(2-3), 111-132. doi:10.1111/j.1540-5834.1995.tb00207.

Tarabulsy, G. M., Moran, G., Pederson, D. R., Provost, M., \& Larose, S. (2011). Adolescent motherhood, maternal sensitivity and early infant development. Dans D. W. Davis \& C. Logdson (Eds.), Maternal Sensitivity: A Scientific Foundation for Practice (pp. 157-178). Haupauge, NY: Nova Publishers.

\section{Pour citer l'article}

Poveda Perdomo, C., Bergeron, C., Yergeau, M., Savage, L.-É., Gauthier-Légaré, A., Lemelin, J.-P., Provost, M., \& Tarabulsy, G. M. (2019). Lien entre le stress parental et la sensibilité maternelle entre six et 18 mois. Psycause: Revue scientifique étudiante de l'École de psychologie de l'Université Laval, 9(2), 19-20. 